\title{
ON ORDINARY RESTRICTED EXTREMA IN CON- NECTION WITH POINT TRANSFORMATIONS
}

\author{
BY JACQUES HADAMARD
}

1. Restricted Minima in a Plane. The question which I shall examine concerns the maximum or minimum of $f(x, y)$, when the variables $x$ and $y$ are not independent, but subjected to the condition

$$
g(x, y)=0,
$$

so that the point $(x, y)$ must describe the curve represented by the preceding equation.

This is what was called, formerly, a relative extremum, but what I proposed to call a restricted ${ }^{*}$ extremum, because the term "relative" is used with another meaning. Indeed, the extrema which we consider, as every extremum treated by the methods of differential calculus, are relative, that is, they are extrema only in comparison with neighboring values of $f$.

The first part of the question, namely, the investigation of stationary values of $f$ on the curve $(g)$, is quite classic. It is usually solved, with the help of the Lagrange multiplier $l$, by means of the simultaneous equations

$$
p+l p_{1}=0, \quad q+l q_{1}=0,
$$

where $p$ and $q$ are the partial derivatives of $f$, and $p_{1}$ and $q_{1}$ are those of $g$. However, we shall use here the condition with $l$ eliminated, that is,

$$
j=p q_{1}-q p_{1}=0,
$$

* The author informs the editors that he, together with David Hilbert and others, had previously agreed that a change of nomenclature is desirable, to replace the older term relative extrema. The agreement is to use "extremum libre ou lie" in French, and "freies oder gebundenes Extremum" in German. The author had used "bound" in English, and the editors have suggested the word "restricted." THE EDITORS. 
so that $j$ is the jacobian of $f$ and $g$. A point $m\left(x_{0}, y_{0}\right)$ of $(g)$ at which $f$ is stationary on $(g)$ necessarily belongs to the curve defined by $(j)$. We shall suppose, throughout this paper, that $m$ is an ordinary point of $(g)$ and of $(j)$. At $m$, the curve $f(x, y)=f\left(x_{0}, y_{0}\right)$ is tangent to $(g)$.

This settles the question of stationary values. But there remains the question whether such a value, when once found, is a maximum or a minimum. This, of course, can be answered easily if we begin by imagining that $x$ and $y$, the coordinates of an arbitrary point of $(g)$, are expressed in terms of a variable parameter $u$. A maximum or a minimum will occur, according to the sign of $d^{2} f / d u^{2}$. Then, expanding the latter derivative and taking account of the fact that

$$
d g=p_{1} d x+q_{1} d y=0, \quad d^{2} g=0,
$$

since the point $(x, y)$ must describe the curve $(g)$, it is easily found that the sign involved is that of

$$
\left(r+l r_{1}\right) q_{1}^{2}-2\left(s+l s_{1}\right) p_{1} q_{1}+\left(t+l t_{1}\right) p_{1}^{2},
$$

where $r, s, t$ denote second derivatives of $f$, and $r_{1}, s_{1}, t_{1}$ denote second derivatives of $g$.

But it is worth while to notice that an equivalent, completely different solution may be obtained by the study of the point transformation

$$
X=f(x, y), \quad Y=g(x, y),
$$

in the neighborhood of $m$.

At $m$ itself, the jacobian $j$ of that transformation vanishes, and the curve $(j$ ) divides the plane (or, more exactly, the region surrounding $m$ ) into two parts $\sigma$ and $\sigma^{\prime}$, the first of which will correspond to $j>0$, the second to $j<0$. Now, we know* that, in the $X Y$ plane, these two regions will have images located on one and the same side of the line $(J)$ which corresponds to $(j)$ : we can say that, in the vicinity of the point $M$, which is the image of $m$, the images of $\sigma$ and $\sigma^{\prime}$ only give one area $R$, limited by $(J)$.

* See my Cours d'Analyse, vol. I. 
In order to solve the given question, we need only inquire whether a direction issuing from $M$ and having $\alpha, \beta$ for its directing cosines, is directed towards the inside of $R$.

Let us call direction parameters of a direction, any two quantities proportional to the direction cosines of that direction, with a positive coefficient of proportionality. In particular, the normal to $(j)$ at $m$, directed towards the inside of $\sigma$, will admit of direction parameters equal to the partial derivatives of $j$,

$$
P=\frac{\partial j}{\partial x}, \quad Q=\frac{\partial j}{\partial y} .
$$

Then let us consider the direction tangent to $(j)$, directed with respect to $\sigma$. It will admit of the direction parameters $Q,-P$, so that an infinitesimal displacement $(d x, d y)$ of $m$ on $(g)$ in the direct sense will satisfy the relations

$$
\frac{d x}{Q}=\frac{d y}{-P}>0 \text {. }
$$

Hence the corresponding displacement in the $X Y$ plane will be such that

$$
\frac{d x}{p Q-q P}=\frac{d y}{p_{1} Q-q_{1} P}>0 .
$$

The latter displacement will be tangent to $(J)$, in the direct sense with respect to $R$.

Therefore a necessary and sufficient condition that a direction issuing from $M$ and not tangent to $(J)$ be directed towards the inside of $R$, is that it shall determine, with the direction defined by (3), an angle with a positive sine. If $\alpha$ and $\beta$ denote the direction cosines of such a direction, this condition may be written in the form

$$
\beta(p Q-q P)-\alpha\left(p_{1} Q-q_{1} P\right)>0 .
$$

The application to restricted maxima or minima of $f$ on the line $(g)$ is obvious. Let us consider an arc of the latter line, which shall contain $m$ and be sufficiently small. Its image in 
the $X Y$ plane will be a portion of a parallel to the $X$ axis, which must lie inside $R$. If we take its origin at $M$, its direction cosines, which are +1 and 0 , must therefore, satisfy the condition (4). We see, then, that the proper sign is plus if we have

$$
p_{1} Q-q_{1} P<0,
$$

and minus if

$$
p_{1} Q-q_{1} P>0 .
$$

In the first case, $X_{0}$ negative, the value of $f$ at $m$, which is the abscissa of $M$, will be a restricted minimum of $f$ under the given conditions; in the second case, a maximum.

Similarly, the curve $f(x, y)=f\left(x_{0}, y_{0}\right)$, which goes through $m$, will have for its image a portion of the straight line $X=X_{0}$, and the direction from $M$ thus defined will be parallel to the positive $Y$ axis or to the negative $Y$ axis, according to the sign of the quantity

$$
p Q-q P
$$

This quantity and the quantity $p_{1} Q-q_{1} P$ have the ratio $-l$, on account of (1). The fact that a restricted maximum of $f$ when $g$ is constant coincides with a maximum or with a minimum of $g$ when $f$ is constant, according to the sign of $l$, is classic.

It is easy to verify by direct computation that the condition thus obtained is the same as that which would result from the expression (2). The remarkable thing is that the latter can be expressed in terms of the first derivatives of $f, g$, and $j$.

2. The Case of a Curve in Space. Let us consider the curves defined by the equations

$$
g_{1}(x, y, z)=0, \quad g_{2}(x, y, z)=0,
$$

and a stationary value, let us say at the (ordinary) point $m\left(x_{0}, y_{0}, z_{0}\right)$, of $f(x, y, z)$ on that curve. 
At $m$, the jacobian

$$
j=\left|\begin{array}{lll}
\frac{\partial f}{\partial x} & \frac{\partial f}{\partial y} & \frac{\partial f}{\partial z} \\
\frac{\partial g_{1}}{\partial x} & \frac{\partial g_{1}}{\partial y} & \frac{\partial g_{1}}{\partial z} \\
\frac{\partial g_{2}}{\partial x} & \frac{\partial g_{2}}{\partial y} & \frac{\partial g_{2}}{\partial z}
\end{array}\right|=\left|\begin{array}{ccc}
p & q & r \\
p_{1} & q_{1} r_{1} \\
p_{2} & q_{2} r_{2}
\end{array}\right|
$$

must vanish. Again, we shall suppose that $m$ is an ordinary point of the surface $j=0$, so that not all the three derivatives

$$
P=\frac{\partial j}{\partial x}, \quad Q=\frac{\partial j}{\partial y}, \quad R=\frac{\partial j}{\partial z}
$$

are zero. Such a surface $(j)$ will divide the space near $m$ into two regions $\omega, \omega^{\prime}$, the first of which will correspond to $j>0$. Let us consider the point transformation defined by

$$
X=f(x, y, z), \quad Y=g_{1}, \quad Z=g_{2} .
$$

Around $M$ (the image of $m$ ), $\omega$ and $\omega^{\prime}$ will have a common image $R$, on one side of the surface $(J)$ which corresponds to $j$. Let us find a necessary and sufficient condition that the direction from $M$ with the direction cosines $\alpha, \beta, \gamma$ be directed towards the inside of $R$. Let $(d x, d y, d z),(\delta x, \delta y, \delta z)$ be two directions from $m$, tangent to $(j)$, so that

$$
\frac{d y \delta z-d z \delta y}{P}=\frac{d z \delta x-d x \delta z}{Q}=\frac{d x \delta y-d y \delta x}{R} .
$$

These two directions, in the above order, will define a direct orientation on $s$, at $m$, if the common value of the above ratios is positive. In the $X Y Z$ space, the corresponding directions will be

$$
\begin{aligned}
d X & =p d x+q d y+r d z, \quad d Y=p_{1} d x+q_{1} d y+r_{1} d z \\
d Z & =p_{2} d x+q_{2} d y+r_{2} d z \\
\delta X & =p \delta x+q \delta y+r \delta z, \quad \delta Y=p_{1} \delta x+q_{1} \delta y+r_{1} \delta z \\
\delta Z & =p_{2} \delta x+q_{2} \delta y+r_{2} \delta z
\end{aligned}
$$


so that the tangent line to $s$ at $M$ will have direction cosines $A, B, C$, such that

$$
\frac{A}{d Y \delta Z-d Z \delta Y}=\frac{B}{d Z \delta X-d X \delta Z}=\frac{C}{d X \delta Y-d Y \delta X} .
$$

Now, our direction $(\alpha, \beta, \gamma)$ will be directed towards $R$ if it gives with (7) a direct trihedron, that is, if

$$
\left|\begin{array}{lll}
d X & d Y & d Z \\
\delta X & \delta Y & \delta Z \\
\alpha & \beta & \gamma
\end{array}\right|>0 .
$$

Such will be the case for a parallel to the positive $X$ axis if

$$
d Y \delta Z-d Z \delta Y>0
$$

or, on account of (6) and ( 7$)$,

$$
P\left(q_{1} r_{2}-r_{1} q_{2}\right)+Q\left(r_{1} p_{2}-p_{1} r_{2}\right)+R\left(p_{1} q_{2}-q_{1} p_{2}\right)>0 .
$$

An analogous solution for extrema on a surface would be interesting to find.

3. Conclusion. Let us come back to the case of the plane. The above argument rests on the properties of the transformation at a point where its jacobian vanishes, which is especially interesting to study from the geometric point of view, as will be done in a paper by Professor Julia to appear shortly in the Bulletin des Sciences Mathématiques. The fundamental fact is that, at such a point $m$, any two curves of the $x y$ plane will, in general, have images which will be tangent to each other; but there is an exception, concerning a certain peculiar direction at $m$. Any curves tangent to that special direction will have ordinarily in the $X Y$ plane, an image that has a cusp, or sometimes a terminal point.* The latter case (a particular case of the former) occurs precisely for our curves $f(x, y)=f\left(x_{0}, y_{0}\right), g(x, y)=0$.

Collège de France

* The words terminal point are suggested by the editors as a translation of the French term point d'arrêt. 